\title{
Ultrasonografía endoscópica para el diagnóstico de áscaris biliar: reporte de caso y revisión de la literatura
}

\section{Endoscopic ultrasound for the diagnosis of biliary ascariasis: case report and literature review}

\author{
Héctor Adolfo Polanía-Liscano, ${ }^{1 *}$ (1) Héctor Conrado Jiménez-Sánchez, ${ }^{2}$ (B] David José Polanía-Galindo., ${ }^{3}$
}

\author{
Gacceso abierto \\ Citación: \\ Polanía-Liscano HA, Jiménez-Sánchez HC, \\ Polanía-Galindo DJ. Ultrasonografía endoscópica \\ para el diagnóstico de áscaris biliar: reporte \\ de caso y revisión de la literatura. Rev Colomb \\ Gastroenterol. 2020;35(4):537-541. https://doi. \\ org/10.22516/25007440.410 \\ Gastroenterólogo clínico quirúrgico. Profesor \\ asociado, Universidad Surcolombiana; Neiva, \\ Colombia. \\ 2 Especialista en Epidemiología Clínica. \\ Cirujano General, Epidemiologo Universidad \\ Surcolombiana. Hospital Universitario Hernando \\ Moncaleano Perdomo; Neiva. Colombia. \\ 3 Médico General Universidad del Rosario; \\ Bogotá, Colombia. \\ *Correspondencia: Héctor Adolfo Polanía-Liscano \\ endotekneiva@gmail.com \\ Fecha recibido: $\quad 30 / 05 / 19$ \\ Fecha aceptado: 15/08/19
}

\begin{abstract}
Resumen
Se presenta un caso de áscaris de la vía biliar $(A B)$, cuya manifestación clínica fue ictericia obstructiva, acompañada de dolor abdominal causado por un cuadro de pancreatitis aguda. Inicialmente, se sospechó de etiología litiásica, por lo cual se realizaron estudios de imágenes diagnósticas y se evidenció la presencia de $A B$ como hallazgo incidental, diagnosticado por ultrasonografía endoscópica biliopancreática (UEB), que fue confirmado y tratado mediante colangiopancreatografía retrógrada endoscópica (CPRE).
\end{abstract}

\section{Palabras clave}

Áscaris de la vía biliar, ictericia obstructiva, dolor abdominal, pancreatitis aguda, etiología litiásica.

\begin{abstract}
The following is a case of biliary ascariasis (BA), whose clinical presentation was obstructive jaundice, accompanied by abdominal pain due to acute pancreatitis. At first, clinical suspicion led to consider a stone etiology, for which diagnostic imaging studies were performed, evidencing BA as an incidental finding diagnosed by endoscopic biliopancreatic ultrasonography (EBU), which was confirmed and treated using endoscopic retrograde cholangiopancreatography (ERCP).
\end{abstract}

Keywords

Biliary ascariasis, Obstructive jaundice, Abdominal pain, Acute pancreatitis, Lithiasic etiology.

\section{INTRODUCCIÓN}

La ascariasis es una enfermedad helmíntica frecuente del tracto gastrointestinal humano, causada por el gusano parasitario Ascaris lumbricoides (AL), y se manifiesta principalmente en países tropicales en desarrollo. La forma adulta de AL, por lo general, permanece en el lumen intestinal sin causar ningún síntoma o problemas de salud significativos. De forma ocasional, el gusano adulto puede migrar al tracto biliar a través de la papila de Vater y originar síntomas similares a la coledocolitiasis o, en escenarios inusuales, puede viajar al conducto pancreático y generar síntomas similares a la pancreatitis.

\section{CASO CLÍNICO}

Paciente masculino de 47 años, proveniente de Leticia (Amazonas, Colombia), quien fue remitido por un cuadro clínico de 2 semanas de evolución consistente en dolor 
abdominal de intensidad 8/10 tipo urente, localizado en el epigastrio e hipocondrio derecho, con irradiación en banda, y asociado a múltiples episodios eméticos de características biliares y la presencia de coluria.

Por esta razón, el individuo consultó a la unidad de salud de II nivel de atención; allí se documentó la existencia de una hiperamilasemia de $595 \mathrm{mg} / \mathrm{dL}$, asociada a hiperbilirrubinemia a expensas de la bilirrubina directa (Tabla 1). Además, se observaron reactantes de fase aguda positivos y hallazgos imagenológicos, mediante tomografía axial computarizada (TAC) contrastada, compatibles con pancreatitis aguda (Balthazar grado D) (Figura 1), la cual fue clasificada como moderada a severa, según el índice de severidad clínico, con un APACHE II (Acute Physiology And Chronic Health Evaluation II) de 7 puntos. En consecuencia, se indicó un manejo a partir de reanimación hídrica, cubrimiento antibiótico con ampicilina/sulbactam y suspensión de la vía oral.

Tabla 1. Reporte de paraclínicos de ingreso y egreso

\begin{tabular}{lcc|}
\hline \multicolumn{1}{c}{ LAB } & Ingreso & Egreso \\
\hline LEU & 7100 & 12000 \\
\hline NTRO & $88 \%$ & $72 \%$ \\
$\mathrm{Hb}$ & $10,7 \mathrm{~g} / \mathrm{dL}$ & $12,2 \mathrm{~g} / \mathrm{dL}$ \\
\hline $\mathrm{FA}$ & $1308 \mathrm{UI} / \mathrm{L}$ & $155 \mathrm{UI} / \mathrm{L}$ \\
\hline BT & $3,61 \mathrm{mg} / \mathrm{dL}$ & $0,75 \mathrm{mg} / \mathrm{dL}$ \\
\hline BD & $2,76 \mathrm{mg} / \mathrm{dL}$ & $0,5 \mathrm{mg} / \mathrm{dL}$ \\
\hline TGO & $233 \mathrm{UI/L}$ & $123 \mathrm{UI/L}$ \\
\hline TGP & $155 \mathrm{UI} / \mathrm{L}$ & $73 \mathrm{UI} / \mathrm{L}$ \\
\hline PCR & $4,8 \mathrm{UI/L}$ & $1,4 \mathrm{UI/L}$ \\
\hline Amilasa & $595 \mathrm{UI} / \mathrm{L}$ & $51,3 \mathrm{UI} / \mathrm{L}$ \\
\hline
\end{tabular}

LAB: laboratorio; LEU: leucocitos; NTRO: neutrófilos; Hb: hemoglobina; FA: fosfatasa alcalina; BT: bilirrubina total; BD: bilirrubina directa; TGO: transaminasa glutámico oxalacética; TGP: transaminasa glutámico pirúvica; $\mathrm{PCR}$ : proteína $\mathrm{C}$ reactiva; UI: unidad internacional

Como estudio complementario, se realizó una ecografía abdominal, en la cual se evidenció la presencia de pólipo vesicular y barro biliar, sin alteraciones a nivel de la vía biliar. Debido al riesgo intermedio de coledocolitiasis, por el aumento de las bilirrubinas totales, la alteración del perfil hepático, la clínica de pancreatitis biliar (Tabla 1) y mayor sensibilidad para microlitiasis, se practicó una ultrasonografía endoscópica que reportó colelitiasis asociada a imagen y fue compatible con AL a nivel de la luz del colédoco, con estructura alargada tubular hiperecogénica, que no daba sombra acústica, e imagen en doble riel (Figura 2). Se indicó un manejo con albendazol (400 mg vía oral, cada 24 h por 3 días) y se efectuó una CPRE, sumada a una papilotomía y a la extracción de áscaris a nivel de la vía biliar, sin complicaciones posoperatorias (Figura 3).

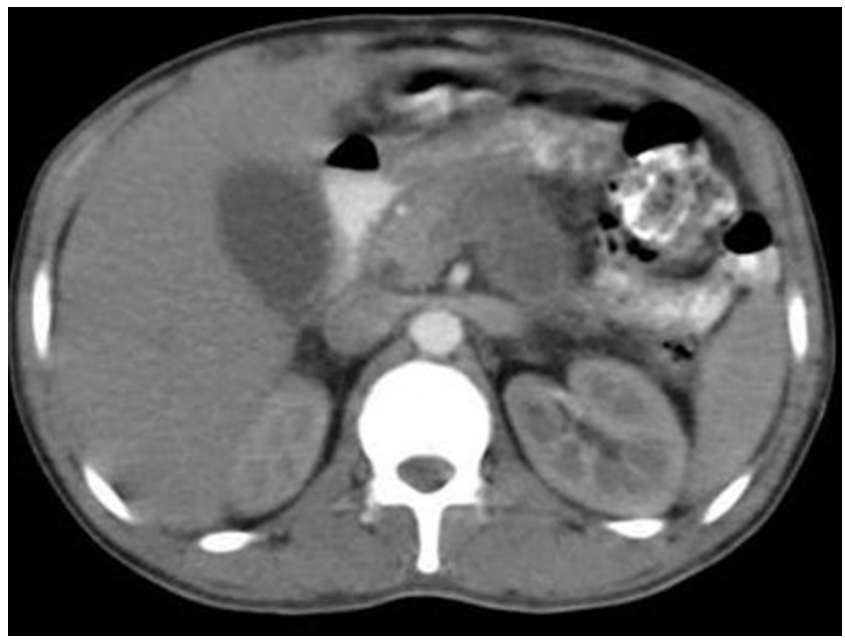

Figura 1. TAC abdominal: hepatomegalia e infiltración grasa, pancreatitis (Balthazar D) con índice de severidad 5, derrame pleural bilateral y escasa cantidad de líquido libre en la cavidad abdominal. Fuente: archivo de los autores.

El paciente presentó una evolución satisfactoria durante la hospitalización, así como la resolución de su pancreatitis aguda. Luego de ello, fue remitido a colecistectomía laparoscópica por causa de la colelitiasis diagnosticada mediante ultrasonografía endoscópica; sin embargo, no se registraron complicaciones.

\section{DISCUSIÓN}

El síndrome ictérico de características obstructivas tiene diversas causas de origen maligno y benigno. Una de ellas —que además es infrecuente y de difícil diagnóstico- es la obstrucción del conducto biliar común (CBC), secundaria a la presencia de $\mathrm{AL}$, que es un nematodo que parasita al humano y puede medir entre 10 y $30 \mathrm{~cm}$ de longitud (1).

En general, cerca del $30 \%$ de los pacientes con hallazgo de AL a nivel del CBC son colecistectomizados. Se ha concluido que dicho antecedente causa una dilatación del $\mathrm{CBC}$, que se asocia con un aumento en la producción de colecistoquinina, lo cual produce la relajación del esfínter de Oddi y facilita la migración del parásito intestinal en el interior del CBC (2).

\section{Epidemiología}

Se han reportado casos en diferentes partes del mundo, en especial en regiones tropicales y subtropicales (África, el 

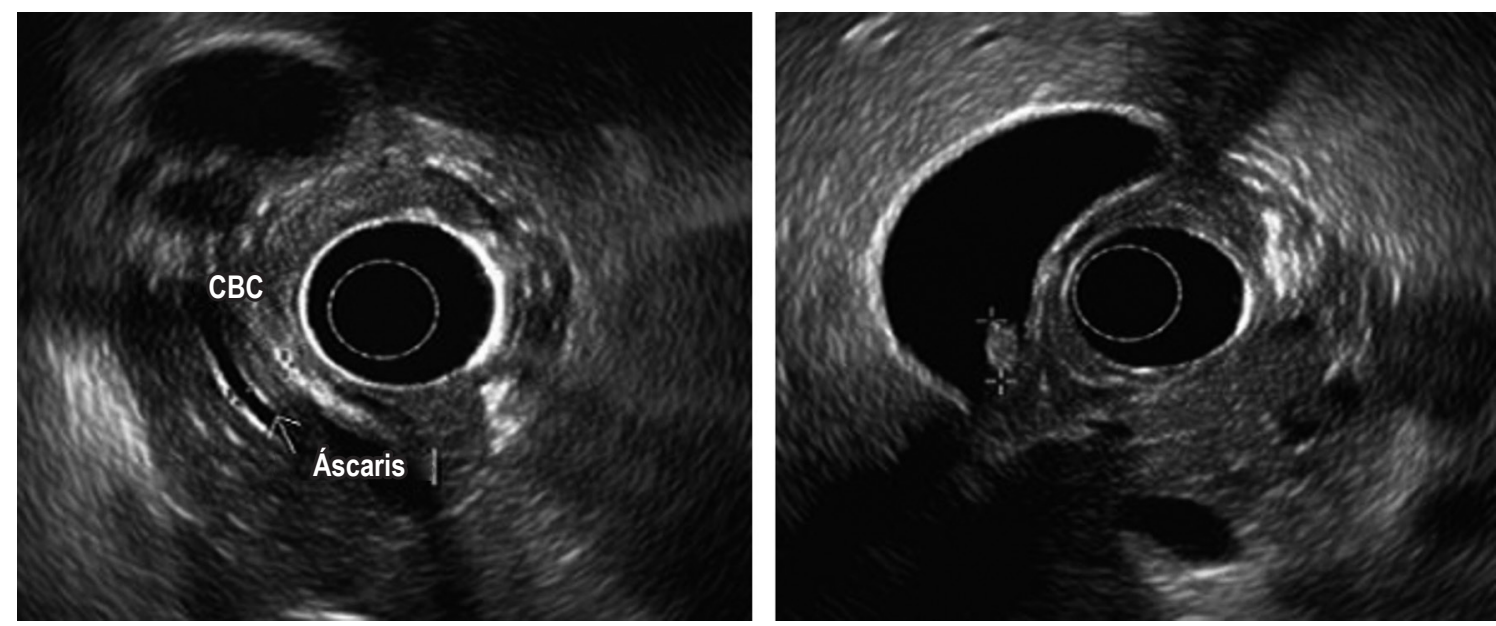

Figura 2. Ultrasonografía endoscópica biliopancreática: colelitiasis, vía biliar con Ascaris lumbricoides en su interior. CBC: conducto biliar común. Fuente: archivo de los autores.
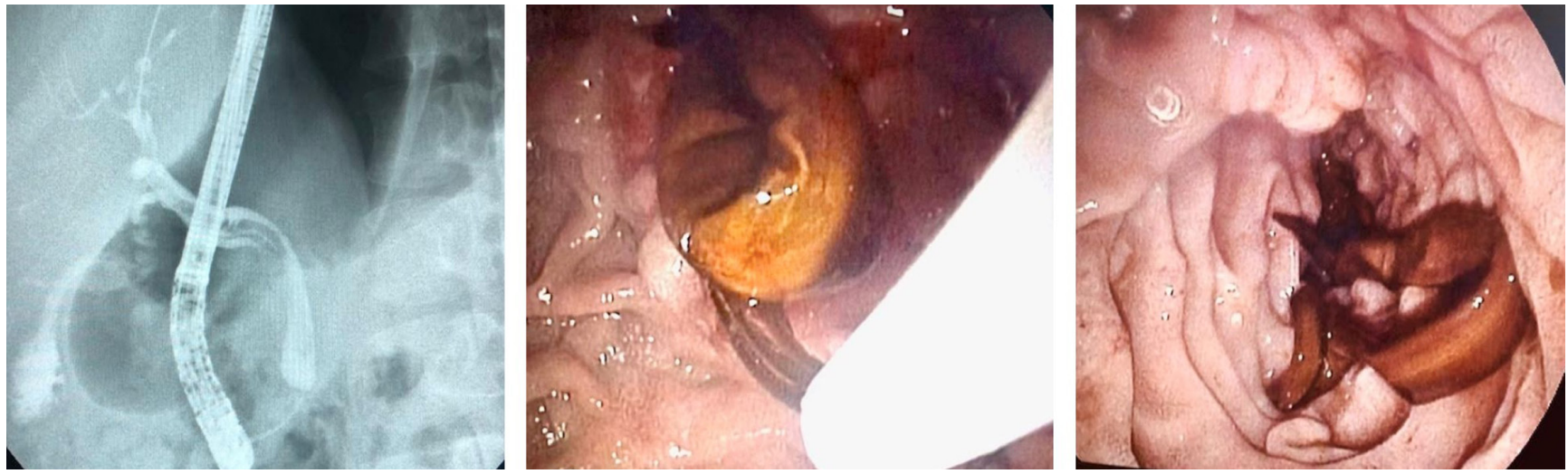

Figura 3. CPRE: vía biliar levemente dilatada de 7 a $8 \mathrm{~mm}$, con defecto de llenado alargado e imagen en doble riel, por presencia de áscaris. Fuente: archivo de los autores.

Lejano Oriente, América Latina, el Sudeste Asiático y algunas partes del Medio Oriente) (3), en donde el suelo y las condiciones climáticas son favorables para la proliferación de las larvas de lombrices intestinales. $\mathrm{El} \mathrm{AB}$ configura el $10-19 \%$ de los ingresos a urgencias relacionados con AL.

No existen datos precisos en relación con la incidencia de $A B$. Sin embargo, se ha documentado una incidencia que oscila entre el 11 y el $19 \%$ en los pacientes con alguna complicación relacionada con el CBC.

\section{Presentación clínica}

\section{Presentación biliar}

Las áreas endémicas, asociadas a pacientes con factores de riesgo para esta entidad, facilitan el diagnóstico de $A B$. Dentro del cuadro clínico se pueden presentar náuseas, vómito, dolor abdominal, ictericia y urticaria. El cólico biliar de intensidad >8-10 (escala análoga del dolor), asociado a colecistitis acalculosa, se presenta en el 56-98 \% de los casos.

Asimismo, dentro de las complicaciones asociadas se menciona la formación de cálculos a nivel del CBC. La colangitis puede estar presente en cerca del $20 \%$ de estos pacientes (4).

No existe una clara relación entre el $\mathrm{AB}$ y el riesgo de colangiocarcinoma. Sin embargo, algunos trabajos describen a este tumor maligno como un factor predisponente que debe considerarse.

\section{Presentación no biliar}

Otra forma de presentación es la pancreatitis aguda secundaria a la ocupación de un AL en la luz del conducto pancreático principal o al nivel del esfínter de Oddi (4-30\% de los casos). 
Además, se puede presentar una respuesta inflamatoria no sistémica, secundaria a la formación de abscesos intrahepáticos, a causa de una alta carga de gusanos a nivel hepático. Este escenario constituye el $1 \%$ de los casos (5).

\section{Diagnóstico}

Con relación a las pruebas de laboratorio, se pueden encontrar tanto una leucocitosis asociada a un aumento del recuento total de eosinófilos como un incremento inespecífico de las pruebas de función hepática. Sin embargo, un mejor indicador es la fosfatasa alcalina, la cual muestra un aumento temprano y progresivo (6) e incluso puede asociarse a la hiperamilasemia, tal y como ocurrió en el caso del paciente descrito.

Existen pruebas inmunológicas que no confirman el diagnóstico de $A B$ (pobres sensibilidad y especificidad). Estas solo son útiles para confirmar la exposición al parásito.

La ecografía hepatobiliar es un método útil para un enfoque diagnóstico inicial, con el típico signo de 4 líneas (CBC con tubo anecoico en su interior). Sin embargo, su sensibilidad oscila entre el 25 y el $86 \%$ (7), y disminuye cuando se suman cuadros de colelitiasis y pancreatitis, e incluso cuando se realiza el estudio en la fase migratoria del gusano hacia el exterior del CBC.

Entre tanto, la colangiorresonancia tiene un rendimiento inferior al $50 \%$ en los casos, debido a la insuficiente delimitación a nivel de la luz del CBC. No obstante, en la secuencia de T2, el parásito puede visualizarse como una estructura hipointensa, con una señal hiperintensa del CBC a su alrededor (8).
De otro lado, estudios previos recomiendan el uso diagnóstico y terapéutico de la CPRE, pues alcanza sensibilidades superiores al $80 \%$. Pese a ello, en los últimos años, por causa del riesgo de complicaciones posquirúrgicas de este método (pancreatitis, sangrado, perforación intestinal), diversos autores han propuesto, en su lugar, la utilización de la ultrasonografía endoscópica como estrategia diagnóstica efectiva para esta entidad. En efecto, la ultrasonografía endoscópica ha demostrado una sensibilidad cercana al $100 \%$, cuando se usa en conjunto con la CPRE $(8,9)$.

Durante el presente caso se realizó, inicialmente, una ecografía hepatobiliar que no entregó datos conclusivos en relación con las características de la vía biliar, mientras que por el riesgo intermedio de coledocolitiasis (10) se practicó una ultrasonografía endoscópica, con el reporte de $A B$ manejado satisfactoriamente mediante CPRE.

\section{CONCLUSIONES}

La ultrasonografía endoscópica biliopancreática es una herramienta diagnóstica de gran utilidad en la actualidad, con altas tasas de sensibilidad y especificidad en las patologías del CBC. Ante la sospecha de una obstrucción biliar de etiología no esclarecida, es necesario evitar intervenciones que presentan morbilidad, mientras que si hay indicios de $\mathrm{AB}$, la ultrasonografía endoscópica biliopancreática constituye el método diagnóstico de elección.

La CPRE combinada con agentes antihelmínticos es un tratamiento efectivo, por lo que se considera de primera línea en el manejo de la $\mathrm{AB}$.

\section{REFERENCIAS}

1. Greenstein AJ, Sachar DB, Gibas A, Schrag D, Heimann T, Janowitz HD, Aufses AH Jr. Outcome of toxic dilatation in ulcerative and Crohn's colitis. J Clin Gastroenterol. $1985 ; 7(2): 137-43$ http://doi.org/10.1097/00004836-198504000-00007

2. Gupta R, Agarwal DK, Choudhuri GD, Saraswat VA, Baijal SS. Biliary ascariasis complicating endoscopic sphincterotomy for choledocholithiasis in India. J Gastroenterol Hepatol. 1998;13(10):1072-1073. http://doi.org/10.1111/j.1440-1746.1998.tb00573.x

3. Leung JW, Chung SC. Endoscopic management of biliary ascariasis. Gastrointest Endosc. 1988;34(4):318-20. http://doi.org/10.1016/s0016-5107(88)71364-4

4. Kamiya T, Morishita T, Reredo R, Marancenbaum C, Montaño C. Duodenoscopic Management in Biliary
Ascariasis. Dig Endosc. 1993;5(2):179-182. http://doi.org/10.1111/j.1443-1661.1993.tb00614.x

5. Pawlowski ZS. Ascariasis. En: Warren KS, Mahmoud AA. Tropical and geographical medicine. Nueva York: Mc Graw-Hill, 2a edición; 1990. p. 369-378.

6. Khuroo MS, Zargar SA, Mahajan R. Hepatobiliary and pancreatic ascariasis in India. Lancet. 1990;335(8704):1503-1506. http://doi.org/10.1016/0140-6736(90)93037-p

7. Khuroo MS, Zargar SA, Mahajan R, Bhat RL, Javid $\mathrm{G}$. Sonographic appearances in biliary ascariasis. Gastroenterology. 1987;93(2):267-272. http://doi.org/10.1016/0016-5085(87)91013-4

8. Nasa M, Patil G, Sharma ZD, Puri R. Biliary Ascariasis Mimicking as Choledocholithiasis on Endoscopic Ultrasound. J Assoc Physicians India. 2019;67(1):81-82. 
9. Sandouk F, Haffar S, Zada MM, Graham DY, Anand BS. Pancreatic-biliary ascariasis: experience of 300 cases. Am J Gastroenterol. 1997;92(12):2264-2267.

10. Aleknaite A, Simutis G, Stanaitis J, Valantinas J, Strupas K. Risk assessment of choledocholithiasis prior to laparosco- pic cholecystectomy and its management options. United European Gastroenterol J. 2018;6(3):428-438.

http://doi.org/10.1177/2050640617731262 